Let $a, b, c$ denote sides of triangle $A B C ; h$ the projection of $c$ on $a ; p, q, r, s$ the sides of the required quadrilateral.

Then

$$
\begin{array}{lll}
q+p=c, \quad s-r=b & & \left(\text { from } A B^{2}\right) \\
q-p=h-a, s+r=h & & \text { (from } \left.B D^{2}\right)
\end{array}
$$

These relations determine $p, q, r, s$ and provide simple constructions for drawing the quadrilateral. The second set (dotted lines) is got by changing the third equation into $p-q=h-a$, so that this set has the same sides as the previous set but in different order.

When $a=h$ we get $p=q$ and the two sets degenerate into one and give Perigal's dissection of Pythagoras' theorem (Mackay's Euclid, 1897, p. 93). When $h=b$ the quadrilaterals become triangles $(r=0)$. When $h<b r$ becomes negative; the construction still holds if the sign convention is admitted.

The construction of the quadrilateral may also be approached trigonometrically. Taking $\theta$ for the angle between sides $s, r$ we find

$$
b \cos \theta+h \sin \theta=c
$$

which determines two values of $\theta$ when $b^{2}+h^{2}>c^{2}$ and one when $b^{\prime \prime}+h^{2}=c^{2}$. Circles could be drawn centrally in $A B^{2}, B D^{2}$ with radii $\frac{1}{2} b, \frac{1}{2}(h-a)$ and tangents to them making $\theta$ with $A B$, etc.

G. D. C. Stokes.

\title{
A new form for the sum of a trigonometric series.
}

1. The usual text book formula for $\Sigma \equiv \sum_{t=0}^{n-1}\left[\gamma^{t} \sin (\alpha+t \beta)\right]$ is $\Sigma=\left[\sin \alpha-\gamma \sin (\alpha-\beta)-\gamma^{n} \sin (\alpha+n \beta)+\gamma^{n+1} \sin (\alpha+\overline{n-1} \beta)\right] / R_{0}$ where $\quad R_{0} \equiv 1-2 \gamma \cos \beta+\gamma^{2}=(1-\gamma \cos \beta)^{2}+\gamma^{2} \sin ^{2} \beta$. Now $\sin \alpha-\gamma \sin (\alpha-\beta)=\sin \alpha(1-\gamma \cos \beta)+\cos \alpha(\gamma \sin \beta)$

$$
=\sqrt{R_{0}} \sin (\alpha+\theta)
$$

where $\quad \theta=\tan ^{-1}[\gamma \sin \beta /(1-\gamma \cos \beta)]$.

Similarly

$$
\begin{array}{r}
\gamma^{n} \sin (\alpha+n \beta)-\gamma^{n+1} \sin (\alpha+\overline{n-1} \beta) \\
=\gamma^{n} \sqrt{R_{0}} \sin (\alpha+\theta+n \beta) .
\end{array}
$$


Hence

$$
\begin{aligned}
\Sigma & =\left[\sin (\alpha+\theta)-\gamma^{n} \sin (\alpha+\theta+n \beta)\right] / \sqrt{R_{0}} . \\
& =-\frac{1}{\sqrt{R_{0}}}\left[\gamma^{t} \sin (\alpha+\theta+t \beta)\right]_{0}^{n} \ldots \ldots \ldots \ldots \ldots . . . \\
& =\frac{1}{\sqrt{\bar{R}_{0}}}\left[\gamma^{t} \sin (\pi+\alpha+\theta+t \beta)\right]_{0}^{n} \ldots \ldots \ldots \ldots \ldots . .
\end{aligned}
$$

where $R_{0}$ and $\theta$ are independent of $n$ and $a$.

By a similar transformation it may be shown that [ ] in (1) is equal to $\sqrt{R_{n}} \sin (\alpha+\theta-\phi)$ where $R_{n} \equiv 1-2 \gamma^{n} \cos \beta+\gamma^{2 n}$ and $\phi=\tan ^{-1}\left[\gamma^{n} \sin n \beta /\left(1-\gamma^{n} \cos n \beta\right)\right]$.

Thus $\Sigma=\sqrt{R_{n} / R_{0}} \sin (\alpha+\theta-\phi)$ a form which might be practically useful if we required a number of separate sums with varying initial angles, but each consisting of $n$ terms; so that $R_{n}$ and $\phi$, as well as $R_{0}$ and $\theta$, would be the same for each sum.

The positive values of the square roots are to be taken. The function $\tan ^{-1}$ being many-valued, a value of $\theta$ must be selected giving $\sin \theta$ the $\operatorname{sign}$ of $\gamma \sin \beta$ and $\cos \theta$ the sign of $1-\gamma \cos \beta$; and similarly $\phi$ will take a value which gives $\sin \phi$ the sign of $\gamma^{n} \sin n \beta$ and $\cos \phi$ the sign of $1-\gamma^{n} \cos n \beta$. It is readily seen that for the sum of the corresponding cosine series we have mereiy to substitute $\cos$ for $\sin$ in the expressions given, the values of $R_{0}, R_{n}, \theta$ and $\phi$ remaining unchanged.

2. In what precedes we have merely transformed a known expression for $\Sigma$, but it is simpler to start $a b$ initio and obtain $\Sigma$ as the inverse of $\Delta$.

Using the transformation of $\$ 1$, with $(\alpha+t \beta)$ for $\alpha$ and $-\beta$ for $\beta$, and therefore $-\theta$ for $\theta$

$$
\begin{aligned}
\Delta_{t}\left[\gamma^{t} \sin (\alpha+t \beta)\right] & =-\gamma^{t}[\sin (\alpha+t \beta)-\gamma \sin (\alpha+\overline{t+1} \beta)] \\
& =-\sqrt{R_{0}} \gamma^{t} \sin (\alpha-\theta+t \beta) \ldots \ldots \ldots \ldots \ldots \ldots
\end{aligned}
$$

where $R_{0}$ and $\theta$ have the values previously found.

Performing $\Sigma$ on both sides

$$
\gamma^{t} \sin (\alpha+t \beta)=-\sqrt{R_{0}} \Sigma \gamma^{t} \sin (\alpha-\theta+t \beta)
$$

whence, putting $\alpha+\theta$ for $\alpha$ and dividing by $-\sqrt{R_{0}}$

$$
-\overline{\sqrt{ } / / R_{0}} \gamma^{t} \sin (\alpha+\theta+t \beta)=\Sigma \gamma^{t} \sin (\alpha+t \beta)
$$


3. The integral $\int e^{\lambda x} \sin (\alpha+\beta x) d x$ is deducible in the form $e^{\lambda x} \sin (\alpha+\beta x-\theta) / \sqrt{\lambda^{2}+\beta^{2}}$ by treating it as a limiting case of the summation cited in $\S 2 . \Delta$ is replaced by $\delta, t$ by $x, \gamma$ by $e^{\lambda}$, and the limits of $\sqrt{R} / \delta x$ and $\tan \theta$ are easily shown to be $\sqrt{\lambda^{2}+\beta^{2}}$ and $\beta / \lambda$.

The method of obtaining this integral in the above form by reversing the derivative process is given by Edwards (Int. Calc. p. 46).

G. J. Lidstone.

\section{To construct an ellipse whose focus $F$ is given, which shall pass through three given points $A, A^{\prime}, A^{\prime \prime}$ (Halley's Problem).}

The following method is applicable to ellipses of small excentricity (such as the orbits of planets) and may be used as an alternative method to finding the directrix which in such cases is situated at a considerable distance.

With centre $F$ describe a circle of any arbitrary and convenient radius $r$. If $F A, F A^{\prime}, F A^{\prime \prime}$ intersect this circle in $a, a^{\prime}, a^{\prime \prime}$, then corresponding chords such as $A A^{\prime}, a a^{\prime}$ intersect on a fixed straight line $D E$ perpendicular to the focal axis of the ellipse, which is in fact the common chord of the two curves and distant $r / e$ from the $S$-directrix. For if $A A^{\prime}$ intersect the directrix in $Z, S Z$ is the external bisector of the angle $A F A^{\prime}$ and is therefore parallel to $a a^{\prime}$. Also $F a: F A=Z D: Z A=$ ratio of perpendiculars from $D$ and $A$ on directrix $X Z$. Hence the perpendicular from $D$ on $X Z=r / e$.

Similarly by taking $A A^{\prime \prime}, a a^{\prime \prime}$ together we get another point $E$ on the common chord which is now completely determined. The line through $F$ perpendicular to $D E$ is the focal axis.

Now let $x$ be any other point on the circle and let $x a^{\prime}$ meet $D E$ in $G$; then the intersection $X$ of $G A^{\prime}$ and $F x$ is a point on the ellipse. It follows then that if the tangent at $a^{\prime}$ meets $D E$ in $T$, $T A^{\prime}$ will be the tangent to the ellipse at $A^{\prime}$. Hence the second 